\title{
Performance of Silicon Photomultipliers in photon number and time resolution
}

\author{
Sergey Vinogradov ${ }^{\mathbf{1}, \mathbf{a}, \mathbf{b}, \mathbf{c}}$ \\ ${ }^{a}$ Department of Physics, University of Liverpool \\ Liverpool L69 7ZE, United Kingdom \\ ${ }^{b}$ Cockcroft Institute of Accelerator Science and Technology \\ Daresbury Sci-Tech, Warrington WA4 4AD, United Kingdom \\ ${ }^{c}$ P.N. Lebedev Physical Institute of the Russian Academy of Sciences \\ Leninsky pr. 53, Moscow 119991, Russia \\ E-mail: Sergey.Vinogradov@liverpool.ac.uk, Vin@lebedev.ru
}

Silicon Photomultipliers (SiPMs) are a novel generation of photon detectors designed as an array of independently operated Geiger-mode APDs (pixels) with common output. SiPM provides proportional detection of low-level light pulses starting from single photons with remarkable photon number and time resolution at room temperature. Now they are worldwide recognized to be competitive with vacuum photomultiplier tubes (PMTs) and avalanche photodiodes (APDs) for scintillation and Cherenkov light detections in such areas as particle physics and nuclear medicine. Well-known specific drawbacks of SiPMs are excess noises caused by stochastic processes of crosstalk and afterpulsing as well as non-linearity and saturation of SiPM response to intense light pulses due to limited number of pixels and noninstant pixel recovery.

This study presents an analysis of SiPM performance based on probability distributions of the key stochastic processes affecting SiPM response: photo-conversion, dark generation, avalanche multiplication, crosstalk and afterpulsing, non-linearity and saturation losses. SiPM performance in photon number (energy) resolution is represented in terms of specific excess noise factors of these processes identified as comparable metrics of their contributions. SiPM time resolution is shown to be defined by photon number resolution and by temporal profiles of photon arrival and photon detection time distributions, and a single electron response.

Analytical results of this approach are applied to compare a performance of the modern SiPMs with each other and with conventional PMTs and APDs in typical scintillation and Cherenkov detection applications. The results also seem to be useful for SiPM characterization, selection, and application-specific optimization as well as for SiPM design improvements.

International Conference on New Photo-detectors

PhotoDet 2015

6-9 July 2015

Moscow, Troitsk, Russia

\footnotetext{
${ }^{1}$ Speaker
} 


\section{Introduction}

Initially Silicon Photomultipliers (SiPMs) have been widely recognized as a new generation of photon detectors due to an exceptional resolution of short weak light pulses. Being designed as an array of independent Geiger-mode APDs (pixels) with a common output, SiPMs generate an output signal as a sum of Geiger breakdowns from each fired pixel producing precisely calibrated charge packets. Thus, this kind of an output represents inherently discrete detection events in an analog form where the number of events or single electron responses (SER) is clearly resolvable. Geiger mode operations of the SiPM pixels also result in good timing resolution of low-level light pulses and complement advantages of SiPM technology in time-of-flight (TOF) applications.

Modeling of a SiPM response and its statistical characteristics was in a focus for many studies even at the early stages of SiPM technology [1]. In contrast with conventional analog APDs and PMTs, an extremely low excess noise factor of charge multiplication mechanism based on Geiger breakdown with strong negative feedback facilitates modeling of a SiPM response in discrete SER units. On the other hand, considerable noise contributions from specific secondary correlated events, namely, crosstalk and afterpulsing processes, make such modeling much more complicated [2]. Another kind of complications is associated with nonlinearity of a SiPM response due to limited number of pixels and non-instant pixel recovering at relatively intense light signals [3].

This report is an overview of analytical models of SiPM response and performance developed and modified with respect to SiPM specifics mostly during last decade. They appear to be reasonable, powerful, and relatively simple tools for analysis of SiPM photon number and time resolution in various applications.

\section{SiPM performance in photon number resolution}

\subsection{Burgess variance theorem and excess noise factor}

Common measure of a detector quality is a signal-to-noise ratio (SNR) defined by stochastic properties of some output signal quantity - a random variable $K$ (e.g. voltage, charge, number of events) - by its mean value $E[K]=\mu_{K}$ and variance $\operatorname{Var}[K]=\sigma_{K}^{2}$ (standard deviation $\sigma_{K}$ ). Inversely, noise-to-signal ratio or resolution $R$ is a common measure of a detector precision in quantifying an incident signal:

$$
R_{K}=\frac{\sigma_{K}}{\mu_{K}}=\frac{1}{S N R_{K}}
$$

Photon detection process combines a series of internal conversions of input photons $N$ into output electrons $K$, and these conversions are random processes. Typically, $N$ is a random variable as well. In order to calculate how the signal resolution changes from an input to an output of a detector, the Burgess variance theorem is applied. Random conversion process is often described as a random amplification of electrons [4]. In order to predict mean and variance of an output random variable $K(N)$ by known mean and variance of an input random variable $N$, amplifications are assumed to be independent on each other, and a mean and variance of an 
output random variable $M$ at non-random input with $N=1$ (single electron) are also to be known as follows:

$$
E[N \equiv 1]=1 \quad \operatorname{Var}[N \equiv 1]=0 \quad E[M \mid 1]=\mu_{M} \quad \operatorname{Var}[M \mid 1]=\sigma_{M}^{2}
$$

By definition, excess noise factor $(E N F)$ of single electron multiplication in this case is

$$
\operatorname{ENF}[M \mid 1]=1+\frac{\sigma_{M}^{2}}{\mu_{M}^{2}}
$$

$E N F$ given by (2.3) is a rather relevant and commonly used measure of a "noisiness" of APDs and PMTs. Accordingly to the Burgess variance theorem, $N$ electrons at the input with known mean and variance

$$
E[N]=\mu_{N} \quad \operatorname{Var}[N]=\sigma_{N}^{2}
$$

give rise to $K$ electrons at the output as follows:

$$
E[K \mid N]=\mu_{M} \cdot \mu_{N} \quad \operatorname{Var}[K \mid N]=\mu_{M}^{2} \cdot \sigma_{N}^{2}+\mu_{N} \cdot \sigma_{M}^{2}
$$

In order to define internal noisiness of this amplification process, we have to consider another definition of $E N F$ typically used in electronics for analysis of amplifier performance, which takes into account an inherently noisy input of the amplifier:

$$
E N F[K \mid N]=R_{K}^{2} / R_{N}^{2}=\frac{\sigma_{K}^{2}}{\mu_{K}^{2}} / \frac{\sigma_{N}^{2}}{\mu_{N}^{2}}
$$

Applying results of (2.5) to (2.6), ENF of noisy input signal amplification is found to be

$$
\operatorname{ENF}(K \mid N)=1+\frac{\sigma_{M}^{2}}{\mu_{M}^{2}} \cdot \frac{1}{\operatorname{Fano}[N]} \quad \operatorname{Fano}[N]=\frac{\sigma_{N}^{2}}{\mu_{N}}
$$

It means that amplification process $E N F$ is sensitive to statistics of input signals: high input noise (super-Poissonian statistics, factor Fano[N] > 1) results in lower ENF masking noisiness of amplification process with respect to single electron amplification, and input with Poissonian statistics (factor Fano[N]=1) does not affect $E N F$ :

$$
\left.\operatorname{ENF}[K \mid N]\right|_{\operatorname{Fano}[N]=1}=\operatorname{ENF}[M \mid 1]
$$

Expression (2.7) allows combining results of step-by-step conversions as a product of specific ENFs resulting in worsening of resolution in each conversion process, and it could be simplified further if factor Fano for some particular process is equal or close to 1:

$$
R_{K}=R_{N} \cdot \sqrt{E N F_{\text {process } 1} \cdot E N F_{\text {process } 2 \cdots}}=R_{N} \cdot \sqrt{E N F_{\text {total }}}
$$

Thus, results of signal detection are completely defined by total $E N F$ of a detection process, and our analysis of SiPM performance in photon number resolution is based on ENF approach (2.9).

\subsection{Specific excess noise contributions}

\subsubsection{Photodetection process}

Conversion of photons in photoelectrons and conversion of photoelectrons in Geiger breakdowns or counts APD are random processes of so-called binomial selection of photons (electrons). A result of a single particular conversion obeys Bernoulli distribution. It is well 
known that binomial selection of Poissonian photons $N$ results in a Poissonian number of counts $K$ with a mean $E[K]=P D E \cdot \mu_{p h}$. An excess noise of the binomial selection process is defined only by statistics of the conversions (Fano[N]=1). It means both expressions (2.3) and (2.7) yield the same result on $E N F$.

Bernoulli process for non-random single photon with detection probability $P D E$ has $E N F_{B e r n}$ :

$$
E N F_{\text {Bern }}=1+\frac{\sigma_{\text {Bern }}{ }^{2}}{\mu_{\text {Bern }}{ }^{2}}=1+\frac{P D E \cdot(1-P D E)}{P D E^{2}}=\frac{1}{P D E}
$$

Binomial selection process for Poissonian photons with mean output number of counts $P D E \cdot \mu_{p h}$ has $E N F_{\text {Binom }}=E N F_{\text {Bern }}=E N F_{P D E}$

$$
E N F_{\text {Binom }}=R_{K}^{2} / R_{N}^{2}=\frac{1}{\mu_{p h} \cdot P D E} / \frac{1}{\mu_{p h}}=\frac{1}{P D E}
$$

\subsubsection{Dark noise}

Dark electron generation is a Poisson process; therefore merging of dark electrons (with mean $\mu_{d a r k}$ ) with photoelectrons (with mean $\mu_{p e}$ ) results in Poisson distribution. As a rule, a mean number of dark counts is used to be subtracted from a mean output counts, however, this subtraction increases an output variance at some fraction $n$ which depends on precision of the subtraction:

$$
E N F_{D C R}=1+\frac{n \cdot \mu_{d a r k}}{\mu_{p e}}=1+\frac{n \cdot D C R \cdot T}{\mu_{p h} \cdot P D E}
$$

Where $n=1$ corresponds to a priory known mean value of DCR, and $n=2$ is a case of equal times being spent for measurements in dark condition and under illumination.

\subsubsection{Multiplication process}

Multiplication process has been considered in details above, and its $E N F_{\text {gain }}$ is independent on statistics of input for Poissonian photoelectrons entering into an avalanche region:

$$
E N F_{\text {gain }}=1+\frac{\sigma_{\text {gain }}^{2}}{\mu_{\text {gain }}^{2}}
$$

However, an output number of electrons produced in this multiplication process follow some probability distribution. In general, we do not need to know this distribution because SiPM provides extremely low $E N F_{\text {gain }} \sim 1.01 \ldots 1.05$ due to strong quenching of an avalanche. Influence of $E N F_{\text {gain }}$ on $E N F$ of the next conversion stage is expected to be weak and it can be evaluated by (2.5)-(2.7), where an output $K$ is re-normalized in SER units as $K_{\text {ser }}$ :

$$
\begin{aligned}
& \mu_{K_{s e r}}=\frac{\mu_{K}}{\mu_{M}}=\mu_{N} \quad \sigma_{K_{s e r}}^{2}=\frac{\sigma_{K}^{2}}{\mu_{M}^{2}} \quad \sigma_{K}^{2}=\mu_{M}^{2} \cdot \sigma_{N}^{2}+\mu_{N} \cdot \sigma_{M}^{2} \\
& \text { Fano }\left[K_{s e r}\right]=\frac{\sigma_{K_{s e r}}^{2}}{\mu_{K_{s e r}}}=\frac{\sigma_{K}^{2}}{\mu_{M}^{2} \cdot \mu_{N}}=\frac{\sigma_{N}^{2}}{\mu_{N}}+\frac{\sigma_{M}^{2}}{\mu_{M}^{2}}=\text { Fano }[N]+\frac{\sigma_{M}^{2}}{\mu_{M}^{2}} \\
& \text { Fano }\left.\left[K_{\text {ser }}\right]\right|_{N-\text { Poissonian }}=1+\frac{\sigma_{M}^{2}}{\mu_{M}^{2}}=E_{N} F_{\text {Gain }}
\end{aligned}
$$


As shown by (2.7) it means that low noise multiplication has a negligible influence on statistics of the next conversions, thus the distribution of primary counts is close to Poissonian.

\subsubsection{Correlated noise: crosstalk and afterpulsing}

Next stages and other types of conversions are associated with crosstalk and afterpulsing effects - secondary Geiger breakdowns initiated by primary ones. Typically, the crosstalk is almost instantaneous responses of pixels surrounding the primary one due to a secondary photon emission from an avalanche area, and the afterpulsing is a delayed response of the same pixel due to de-trapping of avalanche electrons. These correlated stochastic processes result in dramatic changes in statistics of detected events: a number of counts become a non-Poissonian random variable and a time interval between counts deviates from a single-exponential distribution of Poisson processes, as observed, for example, in [5], especially for Hamamatsu MPPCs. R\&Ds of correlated process models, probability distributions, statistical moments as well as associated excess noises are very challenging and highly demanded work in order to utilize a full potential of SiPM technology in a photon-number-resolving detection. Generally, there were a few models proposed to describe correlated processes and probability distribution of number of counts initiated by a non-random single primary one:

1) Binary (only 0 or 1 and no more) secondary counts [6], [7] - Bernoulli distribution;

2) Urn model - binary counts from a fixed number of pixels [8], [9], e.g. adjacent to the primary one (4 side-by-side or 8 with extra corner-by-corner ones) [9] - Binomial distribution;

3) Random single chain or sequence of binary counts - Geometric distribution [2], [10];

4) Branching Poisson process when a primary event, as well as every secondary one, creates the next generation of Poissonian-distributed secondary events [10]-[12]; as shown in [10], this process results in Borel distribution of a total number of events.

In case if SIPM detects a light pulse with a Poissonian number of photons when a total number of detected events obeys a compound Poisson distribution combined by the Poisson distribution of primaries and the specific distribution of secondaries pointed above 1)-4).

Bernoulli distribution is a simplest possible model of correlated event valid only if a probability of the higher-order secondaries is negligible. Binomial model [9] has been developed and applied to describe an optical crosstalk in the case of short-distance absorption of secondary photons (comparable to a pixel pitch). A geometric model has been developed for any kind of correlated events and initially applied for crosstalk with some reasonable results at relatively low values of probability to get one or more correlated events per single primary $P_{\text {corr }}$ $\sim 10 \%-15 \%$. Branching Poisson model initially was focused on a long-distance crosstalk process when all generations of secondaries have not been affected by a limited number of available (ready-to-be-fired) pixels. As verified in several experiments [10], [13], [14], the probability distribution of the crosstalk process agrees fairly well with the branching Poisson process model. However, there is also an observation of depressed high-order crosstalk probabilities with respect to the Borel prediction [15], probably, because of a short-distance crosstalk effect. Recently, an afterpulsing is simulated as a branching Poisson process assuming a single avalanche creating a Poisson-distributed number of afterpulses, and when iteratively applied to each of the generated afterpulses [12]. 
ENF of the correlated processes was initially derived in [2] and discussed [3], and then advanced results were presented in [10].

In case of non-random single primary event initiating some random number of secondary events with probability to get one or more correlated events per single primary $P_{c o r r}$, mean and variance of total number of counts (including a primary one) of Geometric model are found to be

$$
\mu_{\text {Geom }}=\frac{1}{1-P_{\text {Corr }}} \quad \sigma_{\text {Geom }}^{2}=\frac{P_{\text {Corr }}}{\left(1-P_{\text {Corr }}\right)^{2}}
$$

And its $E N F_{\text {Geom }}$ is shown to be as follows:

$$
E N F_{\text {Geom }}=1+\frac{\sigma_{\text {Geom }}^{2}}{\mu_{\text {Geom }}^{2}}=1+P_{\text {Corr }}
$$

Branching Poisson processes are used to be parameterized in a mean number of events in one generation $\lambda$ - the most convenient single parameter of Poisson distribution. A relation between $\lambda$ and $P_{\text {corr }}$ can be easily determined by probability to get zero counts.

$$
P_{\text {Corr }}=1-\operatorname{Pr}(\text { counts }=0)=1-\exp (-\lambda) \quad \lambda=-\ln \left(1-P_{\text {Corr }}\right)
$$

Mean and variance of total number of counts (including a primary one) of branching Poisson model are found to be

$$
\mu_{\text {Bran }}=\frac{1}{1-\lambda} \quad \sigma_{\text {Bran }}^{2}=\frac{\lambda}{(1-\lambda)^{3}}
$$

So, $E N F_{B r a n}$ of the branching Poisson processes is shown to be as follows:

$$
E N F_{\text {Bran }}=1+\frac{\sigma_{\text {Bran }}^{2}}{\mu_{\text {Bran }}^{2}}=\frac{1}{1-\lambda}=\frac{1}{1-\ln \left(1-P_{\text {Corr }}\right)}
$$

Obviously, the branching Poisson process model (2.19) predicts a super-linear dependence of $E N F$ on $P_{\text {corr }}$, much stronger than linear one for the Geometric model (2.16). Expression (2.19) is in a good agreement with some experimental results and Monte Carlo simulations of SiPM response affected by crosstalk, for example, [13], [16]. It also mostly fits the ENF dependence on the mean number of Poisson events in one generation modeled for afterpulsing ([12], Figure 2, except the last point at $\lambda=0.9$ ).

\subsection{Excess noise of nonlinearity}

All processes discussed above are linear conversions: mean number of photons, electrons, counts are in a linear proportion. However, large input signals are converted to output ones with some nonlinearity by any real detector, and a SiPM as an array of binary photon detectors is especially nonlinear. In order to determine a resolution provided by such a detector for an input signal, the detector has to be calibrated (output scale has to be translated to the input scale). Therefore, input signal resolution has to be calculated with a nonlinearity correction [13]:

$$
R_{\text {nonlin }}=R_{\text {out }} \cdot \frac{\mu_{\text {out }}}{\mu_{\text {in }}} / \frac{d \mu_{\text {out }}}{d \mu_{\text {in }}}
$$

On the other hand, it would be convenient to introduce this nonlinear correction in definition of $E N F$ in order to facilitate final estimations of SiPM performance by $E N F_{n o n l i n}$ : 


$$
E N F_{\text {nonlin }}=\frac{R_{\text {nonlin }}^{2}}{R_{\text {in }}^{2}}=\frac{R_{\text {out }}^{2}}{R_{\text {in }}^{2}} \cdot\left(\frac{\mu_{\text {out }}}{\mu_{\text {in }}}\right)^{2} /\left(\frac{d \mu_{\text {out }}}{d \mu_{\text {in }}}\right)^{2}=\frac{\sigma_{\text {out }}^{2}}{\sigma_{\text {in }}^{2}} /\left(\frac{d \mu_{\text {out }}}{d \mu_{\text {in }}}\right)^{2}
$$

Remarkably, that an absolute value of a mean output signal is not included in (2.21) because calibration procedure translates the output mean back to the input mean. So, an internal performance of a detector does not depend on that (while an electronic noise is not accounted).

Obviously, nonlinearity of detectors is caused by lower responsivity for larger signals; however, from statistical point of view nonlinearity is a result of random losses of input photons associated with a new type of specific excess noises.

\subsubsection{Nonlinearity with no pixel recovery: binomial distribution of detected events}

Dynamic range, nonlinearity and saturation of a SiPM response were observed and discussed at early stages of SiPM development, first of all in case of short light pulse detection without recovering of pixels during detection [18]. Initial analytical results on a distribution of a number of fired pixels and photon number resolution of SiPM was presented in [19], [20]. Detailed analysis of photon number resolution and an introduction of an excess noise factor of binomial nonlinearity of SiPM based on (2.20), (2.21) was given in [3]. It shows that nonlinearity makes actual photon number resolution worse while resolution of output signal appears to be better and tends to zero at high pixel load $L$ (mean number of potential detections per pixel) and probability to fire a pixel $P_{\text {fire }}$ :

$$
\begin{aligned}
& P_{\text {fire }}=1-e^{-L} \quad L=\frac{\mu_{p h} \cdot P D E}{N_{p i x}} \\
& \mu_{\text {out }}=N_{p i x} \cdot P_{\text {fire }} \quad \sigma_{\text {out }}^{2}=N_{p i x} \cdot P_{\text {fire }} \cdot\left(1-P_{\text {fire }}\right) \\
& R_{\text {out }}^{2}=\frac{1-P_{\text {fire }}}{N_{\text {pix }} \cdot P_{\text {fire }}}=\left.\frac{1}{N_{p i x}} \cdot \frac{e^{-L}}{1-e^{-L}}\right|_{L \rightarrow \infty} \rightarrow 0 \\
& R_{\text {nonlin }}^{2}=\frac{1}{\mu_{p h} \cdot P D E} \cdot E N F_{\text {nonlin }} \quad E N F_{\text {nonlin }}=\frac{e^{L}-1}{L} \approx 1+\frac{L}{2}+o\left(L^{2}\right)
\end{aligned}
$$

\subsubsection{Nonlinearity with fixed dead time and exponential pixel recovery}

Loss of photon counts due to dead time after a breakdown is a well-known issue for Geiger counters. Initial analysis of a non-paralyzable dead time detection process was made in the 1970s [21], [22]. Based on that results, SiPM photon number resolution and excess noise factor in case of long light pulse detection (pulse width $T>>$ recovery time $\tau$ ) were derived [3].

$$
\begin{aligned}
& \mu_{\text {out }}=\frac{\mu_{p h} \cdot P D E}{1+L \cdot \frac{\tau}{T}} \quad \sigma_{\text {out }}^{2}=\frac{\mu_{p h} \cdot P D E}{\left(1+L \cdot \frac{\tau}{T}\right)^{3}} \\
& R_{\text {out }}^{2}=\left.\frac{1}{\mu_{p h} \cdot P D E} \cdot \frac{1}{1+L \cdot \frac{\tau}{T}}\right|_{L \rightarrow \infty} \rightarrow 0 \\
& R_{\text {nonlin }}^{2}=\frac{1}{\mu_{p h} \cdot P D E} \cdot E N F_{\text {nonlin }} \quad E N F_{\text {nonlin }}=1+L \cdot \frac{\tau}{T}
\end{aligned}
$$


However, exponential pixel recovery due to a recharging of quenching RC circuit was expected to be a better fit to model SiPM performance. This approach predicts higher excess noise due to double randomness of detections: probability to fire pixel and developed gain are both dependent on random time since previous breakdown [23]. ENF, in this case, could be marginally estimated as:

$$
E N F_{\text {nonlin }}=\frac{\left(1+L \cdot \frac{\tau}{T}\right)^{2}}{1+\frac{L}{2} \cdot \frac{\tau}{T}}
$$

\subsection{Photon number resolution and total excess noise factor of SiPM}

Finally, in correspondence with ENF approach discussed in section 2.1 and expressed by (2.9) with nonlinear correction (2.21), performance of SiPM in photon number resolution is defined by total nonlinear ENF - a product of specific ENFs responsible for various noise contributions:

$$
E N F_{S i P M}=E N F_{P D E} \cdot E N F_{D C R} \cdot E N F_{\text {gain }} \cdot E N F_{C T} \cdot E N F_{A P} \cdot E N F_{\text {nonlin }}
$$

However, at least one more noise sources has to be included in the model at the SiPM output - electronic noise of an acquisition circuit - which is additive to the SiPM output noise in such a way that it could be expressed by a corresponding $E N F_{A Q C}$ :

$$
E N F_{A Q C}=1+\frac{\sigma_{A Q C}^{2}}{\sigma_{\text {out }}^{2}} \quad E N F_{\text {total }}=E N F_{S i P M} \cdot E N F_{A Q C}
$$

Thus, SiPM photon number resolution at given resolution of incident photons $R_{p h}$ is

$$
P N R=\left.R_{p h}\left(\mu_{p h}\right) \cdot \sqrt{E N F_{\text {total }}\left(\mu_{p h}\right)} \quad P N R\right|_{\operatorname{Poisson}\left(\mu_{p h}\right)}=\sqrt{\frac{E N F_{\text {total }}\left(\mu_{p h}\right)}{\mu_{p h}}}
$$

\section{SiPM performance in time resolution}

\subsection{Filtered marked point process approach}

Filtered marked point process approach to modeling of a transient response of signal detection and acquisition systems is well-known and developed in-depth [4], [24]. It is based on consideration of a signal as a sequence of point events (photons, electrons) of negligible time duration represented by Dirac delta functions convolved with instrumental response function (SER) of a detector of random amplitudes (marks) and fixed temporal profile $h_{s e r}(t)$. Recently this approach has been applied to SiPM time resolution models with more or less pronounced focus on specifics of the SiPM operations [25], [26], [27]. Point process event is specified by probability of the event in a given time $t$, and probability to initiate instrumental response (to detect photon, to trigger an avalanche) $\rho_{\text {det }}(t)$ is defined by convolution of corresponding probability density functions (PDF) of photon arrival $\rho_{p h}(t)$, primary triggering $\rho_{\text {sptr }}(t)$ (equal to PDF of single photon time resolution histogram), and correlated triggering $\rho_{\text {corr }}(t)$ due to crosstalk and afterpulsing: 


$$
\rho_{\text {det }}(t)=\left[\rho_{p h} * \rho_{\text {sptr }} * \rho_{\text {corr }}\right](t)
$$

SiPM time resolution $\sigma_{\text {time }}$ in case of detection of Poissonian photons by leading edge or constant fraction discriminator technique (output signal crosses the discriminator level Discr at time $t_{\text {Discr }}$ ) is found to be

$$
\sigma_{\text {time }}=\frac{\sqrt{E N F_{\text {SiPM }} \cdot\left[\rho_{\mathrm{det}} * h_{\text {ser }}^{2}\right](t)+\frac{V_{\text {noise }}^{2}}{\text { Discr }^{2}}}}{\sqrt{\overline{N_{p h}}} \cdot \frac{d\left[\rho_{\mathrm{det}} * h_{\text {ser }}\right](t)}{d t}} \mid t=t_{\text {Discr }}
$$

\subsection{Cramer-Rao lower bound of time resolution and total excess time factor}

As soon as $E N F$ appears to be relevant and convenient measure of SiPM performance also in time resolution as a key factor describing time-independent characteristics of SiPM while time-dependent ones are represented by convolutions of temporal profiles, it would make sense to try applying the same formalism (2.21) to detection of time stamps and excess noise of this detection:

$$
\operatorname{ENF}(\text { time })=\frac{\sigma_{\mathrm{det}}^{2}(t)}{\sigma_{p h}^{2}(t)} \cdot \frac{1}{\left(\frac{d<t>}{d<t>}\right)^{2}}=\frac{\sigma_{\mathrm{det}}^{2}}{\sigma_{p h}^{2}}
$$

Where $\sigma_{\text {det }}$ is defined by (3.2), so (without an electronic noise $V_{\text {noise }}$ ) it could be rewritten

$$
\sigma_{\mathrm{det}}^{2}\left(t_{\mathrm{det}}\right)=\left.\frac{1}{N_{p h}} \cdot \operatorname{ENF}\left(N_{p h}\right) \cdot \frac{\left[\rho_{p h} * \rho_{\text {sptr }} * \rho_{\mathrm{sec}} * h_{\text {ser }}^{2}\right](t)}{\left\{d\left[\rho_{p h} * \rho_{\text {sptr }} * \rho_{\mathrm{sec}} * h_{\text {ser }}\right](t) / d t\right\}^{2}}\right|_{t_{\mathrm{det}}}
$$

In the same time, $\sigma_{p h}$ is an incident photon arrival characteristic - an ideal case of the best possible detector performance to be expressed by Cramer-Rao lower bound estimate $\sigma_{C R L B}$ :

$$
\sigma_{p h}^{2}=\sigma_{C R L B}^{2}=\frac{1}{N_{p h}} \cdot \frac{1}{\int_{-\infty}^{\infty} \frac{\left[d \rho_{p h}\left(t-t_{0}\right) / d t_{0}\right]^{2}}{\rho_{p h}\left(t-t_{0}\right)} d t}
$$

Therefore, ratio of real and ideal detector variances (3.3) yields remarkable separation of pure photon number resolving metrics of $\operatorname{ENF}\left(N_{p h}\right)$ and pure time-dependent characteristics of the detector with respect to ideal one (it make sense to define the last factor as an excess time factor, $E T F)$ :

$$
\operatorname{ENF}(\text { time })=\operatorname{ENF}\left(N_{p h}\right) \cdot \operatorname{ETF}\left(t_{\mathrm{det}}, t_{0}\right)
$$

However, these initial thoughts on relations between photon number and time performance as well as a concept of an excess time factor should be further studied in details.

\section{Conclusion}

Analytical modeling of SiPM provides promising results of reasonable simplicity with reasonable attention to SIPM specifics. ENF is found to be the most relevant and universal metrics of SiPM performance. 


\section{Acknowledgments}

This work has been supported in part by the EC under Grant Agreement 329100 "SiPM indepth” and the STFC Cockcroft Institute Core Grant No ST/G008248/1.

\section{References}

[1] D.A. Shushakov and V.E. Shubin, New solid state photomultiplier, in Proc. SPIE, vol. 2397, San Jose, CA: SPIE, 1995, pp. 544-554 [http : //dx . doi . org/10.1117/12 . 206900].

[2] S. Vinogradov et al., Probability distribution and noise factor of solid state photomultiplier signals with cross-talk and afterpulsing, 2009 IEEE Nucl. Sci. Symp. Conf. Rec., pp. 1496-1500, Oct. 2009 http://dx.doi.org/10.1109/NSSMIC. 2009.5402300

[3] S. Vinogradov et al., Efficiency of Solid State Photomultipliers in Photon Number Resolution, IEEE Trans. Nucl. Sci. (2011) 58, p. 9 [http: //dx . doi . org/10 . 1109/TNS . 2010 . 2096474].

[4] H.H. Barrett and K.J. Myers, Poisson Statistics and Photon Counting, in Foundation of Image Science, New York: Wiley, 2004, ch. 11.

[5] P. Finocchiaro et al., Features of silicon photo multipliers: Precision measurements of noise, crosstalk, afterpulsing, detection efficiency, IEEE Trans. Nucl. Sci., vol. 56, no. 3, pp. 1033-1041, Jun. 2009 [http://dx . doi.org/10.1109/TNS . 2009 . 2014308]

[6] P. Eraerds, M. Legré, a Rochas, H. Zbinden, and N. Gisin, SiPM for fast Photon-Counting and Multiphoton Detection., Opt. Express, vol. 15, no. 22, pp. 14539-49, Oct. 2007. [http://Www.ncbi.nlm.nih.gov/pubmed/19550733]

[7] Ramilli, M., Allevi, A., Chmill, V., Bondani, M., Caccia, M. and Andreoni, A., Photon-number statistics with Silicon photomultipliers, J. Opt. Soc. Am. B 27(5), 852-862 (2010). [http://dx.doi.org/10.1364/J0SAB. 27.000852]

[8] I. Afek, A. Natan, O. Ambar, and Y. Silberberg, Quantum state measurements using multi-pixel photon detectors, Phys. Rev. A, vol. 79, no. 4, p. 7, 2009. [http://dx.doi.org/10.1103/PhysRevA.79.043830]

[9] L. Gallego, J. Rosado, F. Blanco, and F. Arqueros, Modeling crosstalk in silicon photomultipliers, Journal of Instrumentation vol. 8 (05) p. P05010, 2013. [http://dx.doi.org/10.1088/1748-0221/8/05/P05010]

[10] S. Vinogradov, Analytical models of probability distribution and excess noise factor of solid state photomultiplier signals with crosstalk, Nucl. Instr. Methods A (2012) 695, pp. 247-251 [http://dx.doi.org/10.1016/j.nima.2011.11.086]

[11] M. Putignano, A. Intermite and C.P. Welsch, Study of the response of silicon photomultipliers in presence of strong cross-talk noise, Proc. IPAC2011, San Sebastián, Spain, pp. 1389-1391, 2011 [http://WWw-linac.kek.jp/mirror/IPAC2011/papers/tupc153.pdf]

[12] A. Para, Afterpulsing in Silicon Photomultipliers: Impact on the Photodetectors Characterization, arXiv:1503.01525v1 [physics.ins-det] 5 Mar 2015, [http://arxiv.org/pdf/1503.01525v1]

[13] A. Gola, A. Ferri, A. Tarolli, N. Zorzi, and C. Piemonte, SiPM optical crosstalk amplification due to scintillator crystal: effects on timing performance, Phys. Med. Biol., vol. 59, no. 13, p. 3615, 2014. [http://dx.doi.org/10.1088/0031-9155/59/13/3615] 
[14] P.W. Cattaneo et al., Development of High Precision Timing Counter Based on Plastic Scintillator with SiPM Readout, IEEE Trans. Nucl. Sci., vol.61, no.5, pp.2657-2666, Oct. 2014 [http://dx.doi.org/10.1109/TNS. 2014.2347576]

[15] A. Biland et al., Calibration and performance of the photon sensor response of FACT - the first GAPD Cherenkov telescope, J. Instrum., vol. 9, no. 10, p. P10012, 2014. [http://dx.doi.org/10.1088/1748-0221/9/10/P10012]

[16] A. Vacheret et al., Characterization and simulation of the response of Multi-Pixel Photon Counters to low light levels, Nucl. Instruments Methods Phys. Res. Sect. A Accel. Spectrometers, Detect. Assoc. Equip., vol. 656, no. 1, pp. 69-83, Nov. 2011. [http://dx.doi.org/10.1016/j.nima.2011.07.022]

[17] A.I. Abramov, Y.A. Kasansky, and E.S. Matusevich, Foundations of Experimental Methods in Nuclear Physics, Second ed., Moscow, USSR: Atomizdat, 1977, ch. 4 (in Russian).

[18] P. Buzhan, B. Dolgoshein, A. Ilyin, V. Kantserov, V. Kaplin, and others, An advanced study of silicon photomultiplier, ICFA Instrum.Bull., vol. 23, pp. 28-41, 2001. [http://WWW.slac.stanford.edu/pubs/icfa/fall01/paper3/paper3.pdf].

[19] A. Stoykov et al., On the limited amplitude resolution of multipixel Geiger-mode APDs, J. of Instrumentation (2007) 2, P06005 [http : //dx . doi . org/10.1088/17480221/2/06/P०6005].

[20] E.B. Johnson et al., New Developments for CMOS SSPMs, 2008 IEEE Nucl. Sci. Symp. Conf. Rec., pp. 1516-1522, Oct. 2008. [ht tp : //dx . doi . org/10 . 1109/NSSMIC . 2008 . 4774701].

[21] W. Feller, An Introduction to Probability Theory and Its Applications. vol. 2, Third ed., New York: Wiley, 1970, ch. XI.

[22] J.W. Müller, Dead-time problems, Nucl. Instr. Methods A (1973) vol. 112, issue 1-2, pp. 45-57 [http ://dx.doi.org/10.1016/0029-554X(73)90773-8].

[23] S. Vinogradov, Probabilistic analysis of Solid State Photomultiplier performance, in proceedings of Advanced Photon Counting Techniques VI, Proc. SPIE (2012) 8375, p. 83750S [http://dx.doi.org/10.1117/12.919971].

[24] H.H. Barrett, R.F. Wagner, K.J. Myers, Correlated point processes in radiological imaging, Proc. SPIE (1997): 3032 pp. 110-124 [http : //dx . doi . org/10.1117/12 . 273975].

[25] S. Seifert, H.T. van Dam, D.R. Schaart, The lower bound on the timing resolution of scintillation detectors, Phys. Med. Biol. (2012) 57 p. 1797. [http://dx . doi . org/10.1088/00319155/57/7/1797].

[26] S. Seifert et al., A Comprehensive Model to Predict the Timing Resolution of SiPM-Based Scintillation Detectors: Theory and Experimental Validation, IEEE Trans. Nucl. Sci. (2012) 59 p. 190 [http : //dx . doi.org/10.1109/TNS . 2011. 2179314].

[27] S. Vinogradov, Analytical model of SiPM time resolution and order statistics with crosstalk, Nucl. Instr. Methods A (2015), pp. 229-233 [http://dx.doi.org/10.1016/j.nima.2014.12.010]. 